Research Article

\title{
All-Optical Surface Micropatterning by Electric Field Intensity Gradient
}

\author{
U. Gertners and J. Teteris \\ Institute of Solid State Physics, University of Latvia, 8 Kengaraga, Riga LV-1063, Latvia \\ Correspondence should be addressed to U. Gertners; gertners@gmail.com
}

Received 4 June 2015; Revised 15 August 2015; Accepted 31 August 2015

Academic Editor: Jung Y. Huang

Copyright ( 2015 U. Gertners and J. Teteris. This is an open access article distributed under the Creative Commons Attribution License, which permits unrestricted use, distribution, and reproduction in any medium, provided the original work is properly cited.

In this report an all-optical photo-induced formation of surface relief gratings is shown. For the surface patterning of $\mathrm{As}_{2} \mathrm{~S}_{3}$ and $\mathrm{As}_{4} \mathrm{~S}_{1.5} \mathrm{Se}_{4.5}$ films a direct holographic recording setup with a $532 \mathrm{~nm}$ wavelength $N d$ :YAG CW laser light was used. Our investigations have shown that the light-induced mass transfer process strongly depends on the material itself and on the polarization of the light. It has been shown that an electric field intensity gradient has to be obtained to achieve a direct patterning. The evolution of a surface relief in relation to recording parameters and thickness of the sample has been investigated in detail.

\section{Introduction}

A fundamental research of all-optical or direct recording techniques provides a wide range of possible applications, such as the nano-/micropatterning of surfaces, as well as applications for the production of various-purpose optical elements. The demand for lower cost surface relief based optical instruments such as grating-based resonators or filters for waveguides, diffractometers, spectrometers, and so forth is one of the main driving forces for the investigation of a direct light induced relief formation. The complete development of this method would open a wide range of possibilities in the production and utilization of optical element and would introduce new and innovative technologies. The obtained micro- and nanosurface patterns have already been demonstrated as optical polarizers [1], angular or spectral filters $[2,3]$, optical transmission devices [4], data storage devices [5], and even photonic crystals [6-9]. They are also used in the production of tunable lasers, where the wavelength can be varied and depends on the geometrical parameters of gratings $[10,11]$. A number of scientific books have been written on this subject [12-15]. These are just a few of the possible options that could be gained or that could be improved with this new all-optical surface patterning technique. Additionally this processing technique will continue to improve the performance of materials in existing applications and will open the door to new materials and novel applications that would not be easily achievable through other means.

\section{Materials and Methods}

Thin films of amorphous $\mathrm{As}_{2} \mathrm{~S}_{3}$ and $\mathrm{As}_{4} \mathrm{~S}_{1.5} \mathrm{Se}_{4.5}$ with different thickness $(0.5-10.0 \mu \mathrm{m})$ were prepared by thermal deposition on the glass substrates. The pressure during evaporation was $10^{-6}$ Torr and the deposition rate was $20 \mathrm{~nm} / \mathrm{s}$. The thickness of films was controlled by optical interferometer during the deposition. The experiments of surface relief grating formation were performed using a holographic recording system (Figure 1) where a $532 \mathrm{~nm} \mathrm{Nd:YAG} \mathrm{CW}$ laser was used as the irradiation source. The following configurations of holographic recording were applied: a recording from the film-side of the samples (the most popular condition) and a recording from the substrate-side (light penetrates through the substrate before it reaches the film). It is possible to acquire up to four-beam recording setup by slightly modifying holographic recording scheme with additional two side-beams (i.e., all light sources aligned to a circle). These kinds of multibeam recording experiments also were performed. The grating period of $\Lambda=1 \mu \mathrm{m}$ was used in all experiments. The recording efficiency was controlled by measuring the intensity of the 1st-order diffracted beam 


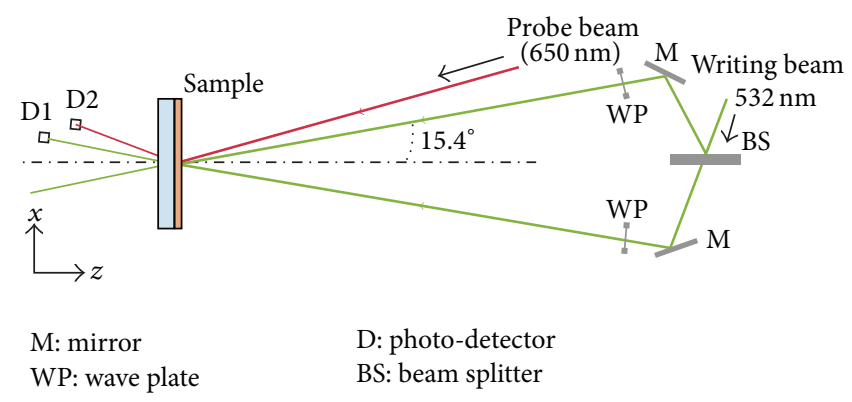

FIGURE 1: Experimental setup for direct holographic recording.

(reflected) of $405 \mathrm{~nm}$ light as well as the intensity of the 1st-order diffracted beam (transmitted) of $650 \mathrm{~nm}$ light. The diffraction efficiency ( $\eta$ or DE) of the holographic gratings is defined as $\eta=P_{\text {dif. }} / P_{0} \cdot 100$, where $P_{\text {dif. }}$ is the intensity of the first-order diffracted beam and $P_{0}$ is the intensity of the incident beam ( 650 or $405 \mathrm{~nm}$ ). The film thickness and large surface reliefs were determined by profilometer Veeco Dektak 150. A high resolution optical microscope NIKON ECLIPSE L150 and atomic force microscope Veeco CP-II were used to assess visual characteristics of the film and to study the surface topography. The theoretical plane wave interference intensity and its polarization distribution have been obtained, therefore significantly simplifying the analysis of the experimental data.

\section{Results and Discussion}

3.1. A Typical All-Optical Holographic Recording Process. In the typical direct holographic recording process the transmission and reflection diffraction efficiency (DE or $\eta$ ) changes with time for amorphous chalcogenide semiconductor films (Figure 2). The obtained gratings are shown in Figure 3. For this particular case the recording was performed on the $\mathrm{As}_{2} \mathrm{~S}_{3}$ sample with a $p$ polarization $\left(I_{1}=I_{2}=0.2 \mathrm{~W} / \mathrm{cm}^{2}\right)$. As photo-induced changes of absorption and refractive index in the resist material are comparatively fast, the transmission DE (dashed curve in Figure 2) reaches maximum ( $55 \%$ DE) very quickly. Due to the overexposure the contrast of the volume grating decreases; transmission DE starts to decrease. At this particular time the reflection DE (solid curve in Figure 2) starts to increase linearly, which is an evidence of the surface relief formation. The small peak for the reflection DE curve is related to the photo-induced reflectivity and changes in the refractive index within the material. From the literature and our experience we know that such direct recording process is reversible for the amorphous chalcogenide monolayer $[16,17]$. The reversibility factor is a vital stage, because the further work is based on the research of mass transfer processes in amorphous chalcogenides.

\subsection{A Simple Method for Estimation of the Optimal Film Thick-} ness. The direct recording process is described by measuring the development of DE during the recording process and its shape or slope $(\Delta \eta / \Delta t)$ coefficient can be used as relative comparison criteria for the recording efficiency. For simplicity,

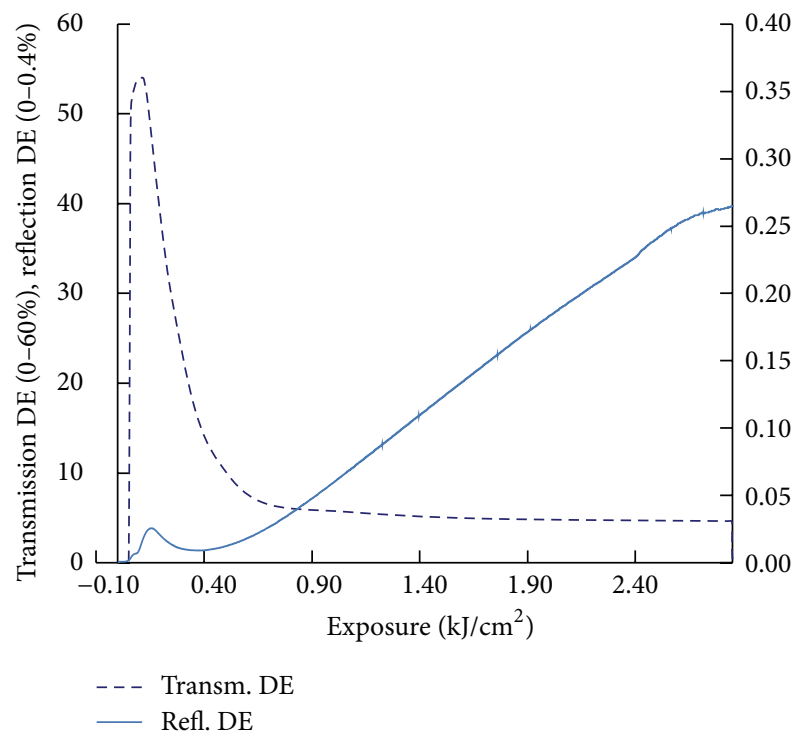

FIGURE 2: Typical $p$ polarization holographic record process is shown by measuring transmission DE $(650 \mathrm{~nm})$ and reflection DE $(405 \mathrm{~nm})$ in real time.

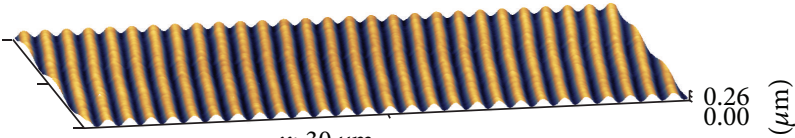 \\ $x: 30 \mu \mathrm{m}$}

FIgURE 3: Obtained hologram in amorphous $\mathrm{As}_{2} \mathrm{~S}_{3}$ thin film, grating period $\Lambda=1 \mu \mathrm{m}$.

DE slope coefficients will be mentioned in $10^{-7} \mathrm{sek}^{-1}$ units or just as arbitrary units. The dependence of DE slope coefficients on the film thickness for the samples illuminated from the film-side as well as from the glass substrate-side is shown in Figure 4(a). Exactly the same experimental results are shown in Figure 4(b); only in this case the profile height $\Delta h$ was obtained by an AFM for each record. From the graphs in Figures 4(a) and 4(b), it can be seen that the DE slope coefficient curves for different thicknesses of the sample describe the obtained surface heights very accurately. In the range of sample thickness up to $2 \mu \mathrm{m}$ the dependence of the profile depth on the film thickness at an equal exposition does not differ essentially for the samples illuminated from 


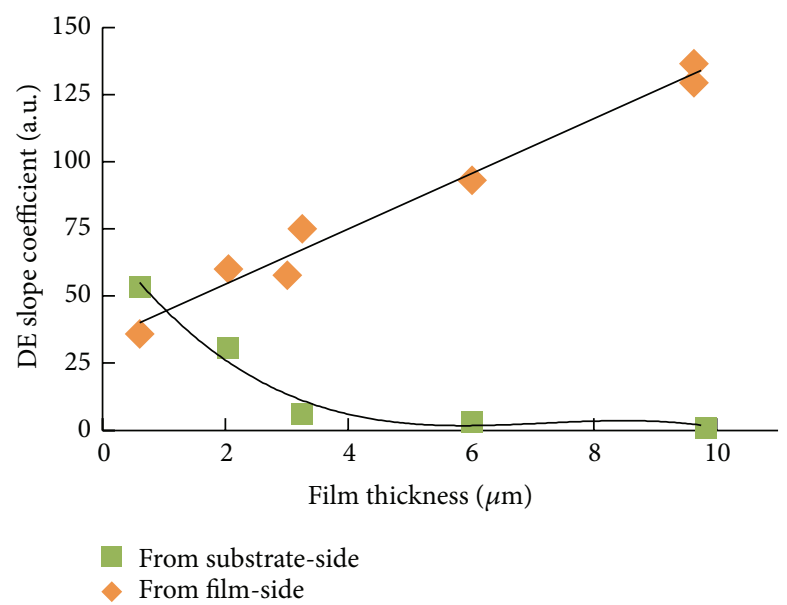

(a)

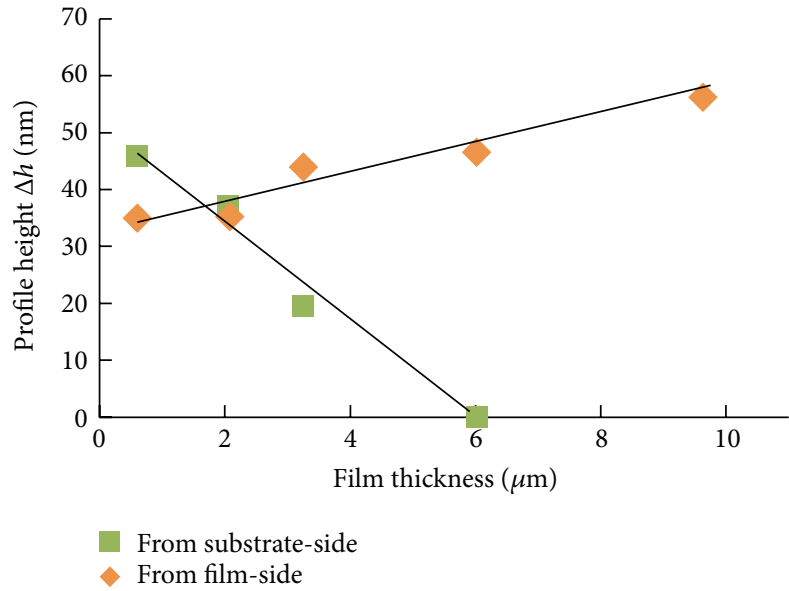

(b)

FIGURE 4: (a) Relative comparison of reflection DE slope coefficient for different $\mathrm{As}_{2} \mathrm{~S}_{3}$ sample thickness of the record from the film-side and from the substrate-side and (b) obtained profile height $\Delta h$ comparison in both of these cases. 45 and -45 polarization holographic recording were performed by a $532 \mathrm{~nm}$ wavelength $\left(I_{1}=I_{2}=0.25 \mathrm{~W} / \mathrm{cm}^{2}\right.$, recording time $t=1 \mathrm{~h}$, and period $\left.\Lambda=1 \mu \mathrm{m}\right)$.

the film- or the glass substrate-side. Thus it is possible to determine the active recording depth or the optimal film thickness, which in this case is approximately $2 \mu \mathrm{m}$. This value describes the recording setup as a whole unit and it depends on all recording parameters; therefore this value has to be determined individually for each recording setup.

In the case of recording through the glass substrate the value of $\Delta h$ decreases rapidly with increasing the film thickness. For the films with a thickness $d>6 \mu \mathrm{m}$ the profile changes were not observed. The calculation of optical absorption coefficient at a recording wavelength $532 \mathrm{~nm}$ for the amorphous $\mathrm{As}_{2} \mathrm{~S}_{3}$ film in a photodarkened state produced a value of $\alpha \approx 10^{4} \mathrm{~cm}^{-1}$. It means that the penetration depth of recording light in the film is about $1 \mu \mathrm{m}$; that is, each onemicron thick layer will absorb the light Euler's number times (Lambert-Beer's law, Euler's number $e \approx 2.7$ ). This explains the fact that for a thin sample the recording efficiency does not differ essentially for the samples illuminated from filmor glass substrate-side. Moreover, the recording through the glass substrate at this point slightly improves the recording efficiency. If the phenomenon of the surface relief formation is a process within the bulk, then the following increment of film thickness will not affect the formation of surface features during the recording from the film-side; meanwhile it will have a significant influence on this recording process from the opposite side of the sample.

3.3. Recording Efficiency versus the Polarization State of the Holographic Setup. Table 1 shows the summary of recording efficiency with several interfering radiation polarization combinations. After the evaluation of direct recording, which is based on the slope coefficient values, we can see that the direct surface patterning highly depends on the selected polarization. It is shown that outstanding direct recording efficiency can be reached with orthogonally \pm 45 polarized light beams. In this case the recording efficiency or the DE
TABLE 1: Direct surface patterning possibilities in amorphous $\mathrm{As}_{2} \mathrm{~S}_{3}$ thin films by using direct holographic recording technique. Recording was performed from the film-side by a $532 \mathrm{~nm}$ wavelength light $\left(I_{1}=I_{2}=0.25 \mathrm{~W} / \mathrm{cm}^{2}\right.$, recording time $t=1 \mathrm{~h}$, and period $\Lambda=$ $1 \mu \mathrm{m})$.

\begin{tabular}{lcc}
\hline $\begin{array}{l}\text { Polarization of } \\
\text { the light }\end{array}$ & $\begin{array}{c}\text { Recording efficiency } \\
(\Delta \eta / \Delta t), 10^{-7} / \text { sek. }\end{array}$ & $\begin{array}{c}\text { Relative comparison } \\
\text { of direct record }\end{array}$ \\
\hline$p$ and $p$ pol. & 0.35 & Good \\
$s$ and $s$ pol. & 0.1 & Poor \\
$s$ and $p$ pol. & 0.04 & Very poor \\
45 and -45 pol. & 44 & Very good \\
\hline
\end{tabular}

slope coefficient is at least two orders of magnitude higher in comparison to other polarization combinations. In fact, $s$ and $p$ polarization setup has the same orthogonally polarized light beams but the efficiency of the relief formation is the worst. In order to continue data analysis and determine the divergence of the given results, it is necessary to carry out more complicated experiments. The next sections will describe the reasons for these results.

\subsection{Theoretical Calculations of the Intensity Distributions and} Their Relation to the Experimental Data. In order to facilitate the interpretation of experimental results and analysis, this part describes in theory all the most popular types of the electric field intensity distributions versus the state of recording beam polarization. The scheme for these theoretical experiments is chosen the same as it was for the recording experiments discussed above. The Bragg equation defines the angle $2 \alpha$ between the corresponding $\mathbf{k}$-vectors for $1 \mu \mathrm{m}$ interference period $\Lambda$ by using a $532 \mathrm{~nm}$ light:

$$
\Lambda=\frac{\lambda}{2 \sin \alpha} \text {. }
$$




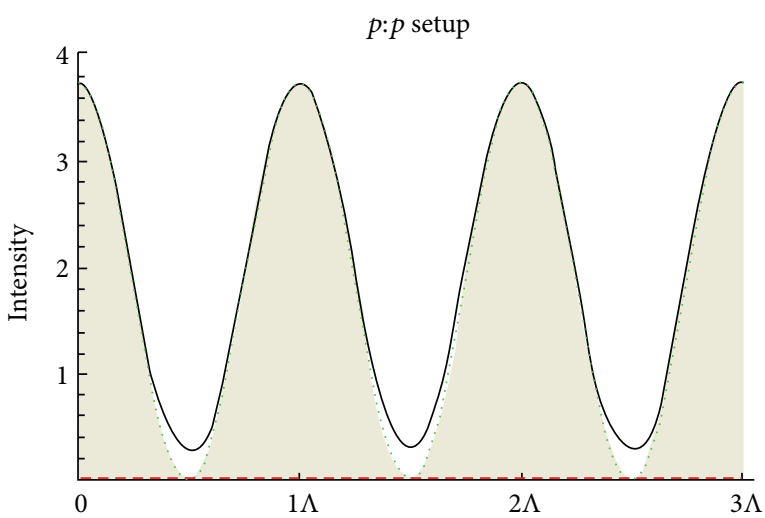

(a)

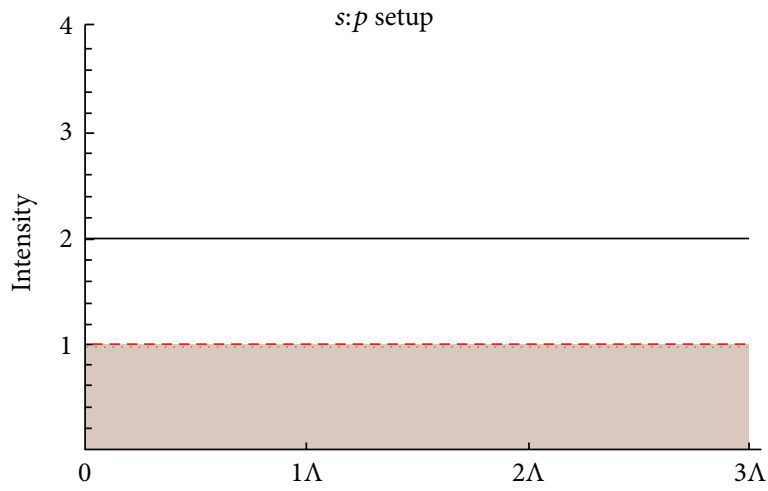

(c)

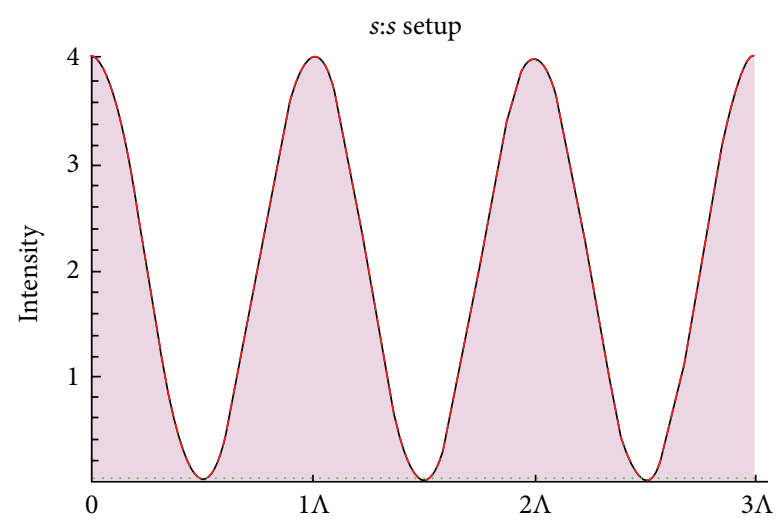

(b)

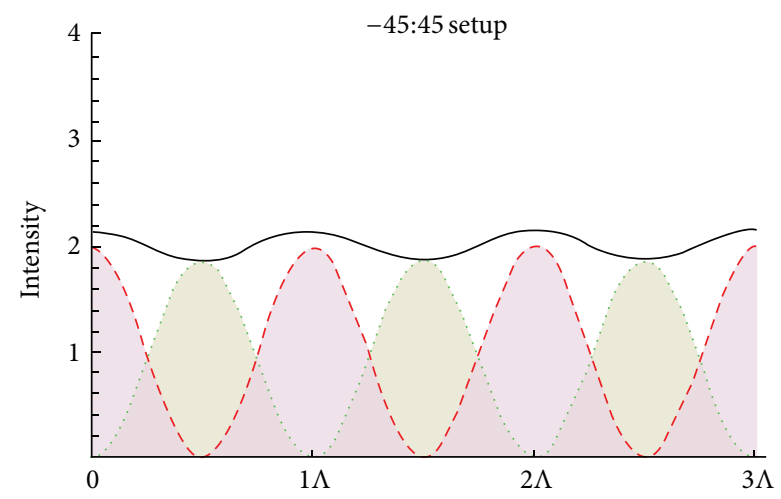

(d)

FIgURE 5: Theoretical light intensity distributions (solid curves) for two coherent beams interference; dashed and dotted curves correspond to the $s$ and $p$ polarized electric field intensity components, respectively. Intensity of the interfered light $I_{1}=I_{2}=1$ unit, $\Lambda$-period, angle between the corresponding $\mathbf{k}$-vectors $2 \alpha=30.9^{\circ}$, period $\Lambda=1 \mu \mathrm{m}$, and $\lambda=532 \mathrm{~nm}$.

By taking solution for a scalar wave equation in a homogeneuos medium,

$$
u=E \cdot \cos (k \cdot s-\omega \cdot t),
$$

where $E$ is amplitude; $k$ is wave number; $s$ is one-dimensional coordinate; $\omega$ is angular frequency; $t$ is time. It is possible to define a vertical or in the $y / z$ coordinate plane polarized wave that propagates along $y$-axis:

$$
\vec{w}=\{0, s, u\}
$$

and horizontal or in the $x / y$ coordinate plane polarized wave that propagates along $y$-axis:

$$
\vec{w}=\{u, s, 0\} .
$$

Together with the coordinate transformation matrix and a data visualization program it is possible to build a theoretical model of the polarization dependent interference.

Figure 5 shows theoretically calculated polarization distributions of two types of plain wave interference. For the simplicity, a colon is used to denote a polarization combination. The black curves represent a total intensity of the interference. Two of the intensity distribution components are shown separately; dotted and dashed curves (and the masks under them) correspond to the intensity from the electric field parallel ( $p$-direction) and perpendicular $(s-$ direction) to the plane of incidence or to the grating vector, respectively. There is also the third component for intensity distribution and that is with direction orthogonal to the $s$ and $p$-direction but that is comparatively small and is not pointed out.

By comparing the obtained theoretical two-beam interference distributions (Figure 5) with previously obtained record efficiency or DE slope coefficient (Table 1), it can be concluded that an effective recording ( \pm 45 polarizations) does not require a large light intensity gradient. The absence of the gradient, like in the case of $s: p$ polarization, also does not guarantee an effective recording. If we look closer to these two cases then everything becomes clear; the most promising light distribution for the surface patterning consists of periodic $s$ and $p$ electric field components, and both intensity distributions have been shifted in respect to one another by a half-period. Thus, the $s$ and $p$ polarized electric field intensity gradients help each other to create a contraction of the film or a formation of valley and an expansion of the film or a formation of a ridge at the same time. On both of the periodic electric field intensity gradients the matter interacts 


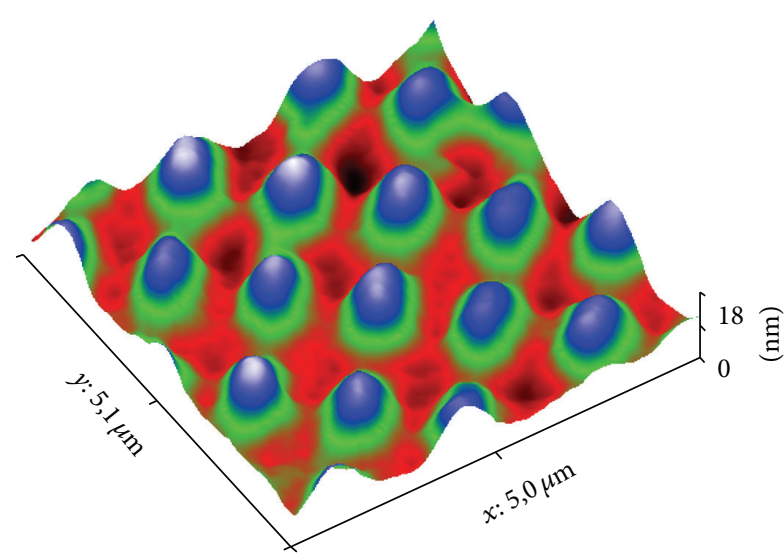

(a)

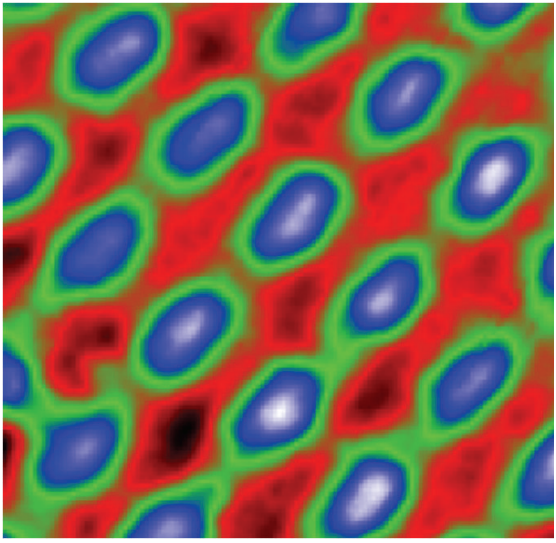

(b)

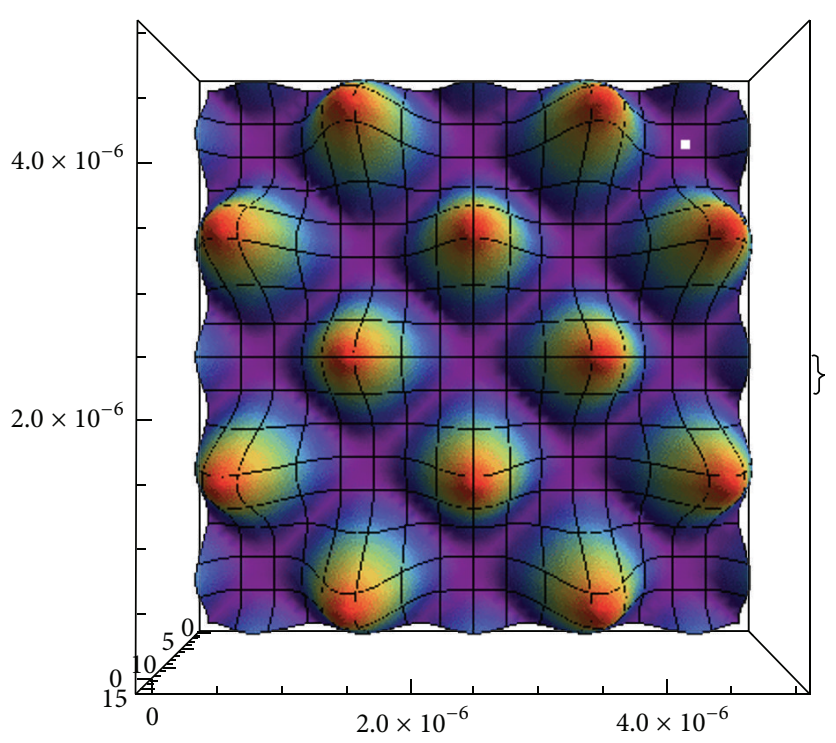

(c)

Figure 6: (a, b) AFM pictures of four-beam holographic recording in $\mathrm{As}_{4} \mathrm{~S}_{1.5} \mathrm{Se}_{4.5}$ sample and (c) its comparison with the theoretical light interference intensity distribution. In this case all four beams are with the same polarization direction.

differently; the lateral mass transfer occurs parallel to the electric field intensity gradient and the polarization of the gradient determines precisely in which direction. If just one $s$ or $p$ component of the electric field intensity gradient is used ( $s: s$ or $p: p$ setup), then the recording efficiency significantly decreases.

The surface relief formation has also been observed with a direct multibeam holographic recording setup (Figures 68). The theoretical intensity distribution has been obtained for each of the setups. First we look at the four-beam setup (Figure 6). If all light sources are polarized in the same direction then the light interference contains highly expressed peaks (16 times more intense than each of the falling light beams) and at the minimum the intensity of light is equal to zero. In this case we can see that the obtained surface relief repeats the theoretically calculated intensity distribution. If the polarization of two opposite light beams is rotated by 45 degrees, then the light intensity gradient is reduced; thus the intensity peaks are not as high and the intensity at the minimum also is no longer equal to zero (Figure 7). When comparing this distribution with the experimental data it can be seen that also in this case the mass is transferred to the light-intensive areas. An identical situation is observed in three-beam holographic recording when all three sources of light are polarized in one direction (Figure 8); the relief repeats the theoretical interference intensity distribution.

With multibeam (three- and four-beam) holographic recording equipment it is possible to obtain different types of 3D point intensity distributions and thus also record various 3D structures. Such dot-shaped intensity distributions are attractive because high-precision structures can be easily recorded in one stage using precalculated interference intensity distributions.

\subsection{Photo-Induced Dielectrophoretic Model of Direct Surface} Relief Formation. It is known that molecules and nanoparticles can be manipulated in fluidic systems using dielectrophoretic (DEP) forces [18]. A DEP force is generated when 


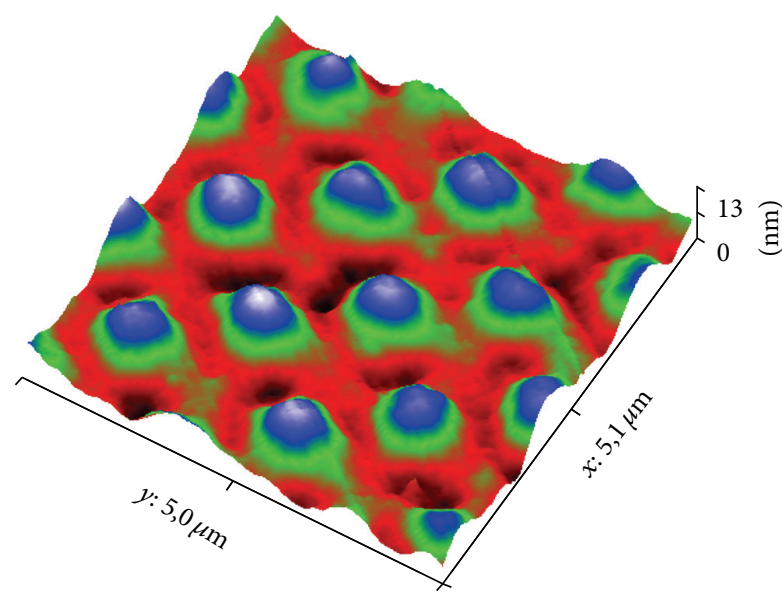

(a)

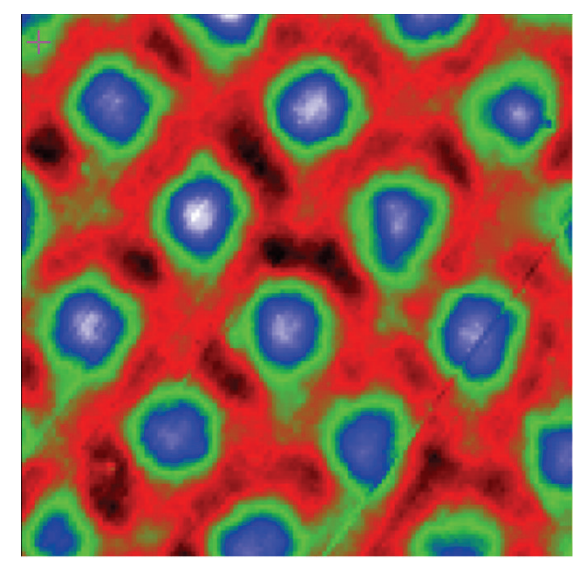

(b)

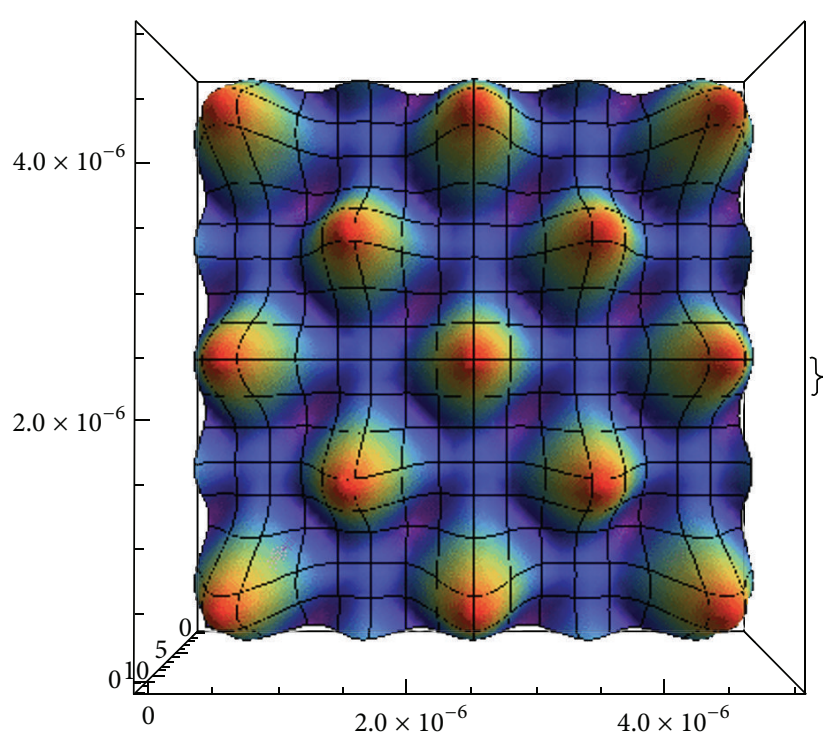

(c)

FIGURE 7: (a, b) AFM pictures of four-beam holographic recording in $\mathrm{As}_{4} \mathrm{~S}_{15} \mathrm{Se}_{4.5}$ sample and (c) its comparison with the theoretical light interference intensity distribution. In this case of two opposite light beams polarization is turned by 45 degrees.

a neutral molecule or particle is suspended in a nonuniform electric field. This electric field induces electrical charges within the particle to establish a dipole. If a nonuniform electric field is applied, the ends of the dipole experience unequal Coulomb forces, which result in a total nonzero imposed force. If the particle is less polarizable than the suspending medium, it is repelled from the regions of higher electric field and the motion is called negative dielectrophoresis while the opposite case is referred to as positive dielectrophoresis.

We propose a photo-induced DEP model to explain the photo-induced mass transport in amorphous films. Model is based on the photo-induced softening of the matrix, formation of defects with enhanced or decreased polarizability, and their drift under the electrical field intensity gradient of the light. An interference pattern from the orthogonal \pm 45 degree polarization can be expressed as a sum of two linearly polarized light components: the electric vector parallel to the grating vector or the $p$-polarization and the electric vector perpendicular to the grating vector or the $s$-polarization (Figure 5(d)). For both components the electric field intensity gradient is parallel to the grating vector. This polarization modulation causes an arrangement of polar photo-induced defects in the film. As a result their polarizability regarding the direction of electric field intensity gradient has been increased by $p$-electric field component and decreased by $s$-electric field component. Due to the DEP forces material is transferred out of the $s$-polarized light region into the $p$ polarized region.

\section{Conclusions}

It has been determined that the highest possible efficiency of the all-optical surface relief holographic recording can be achieved using \pm 45 -degree polarization. This combination of 


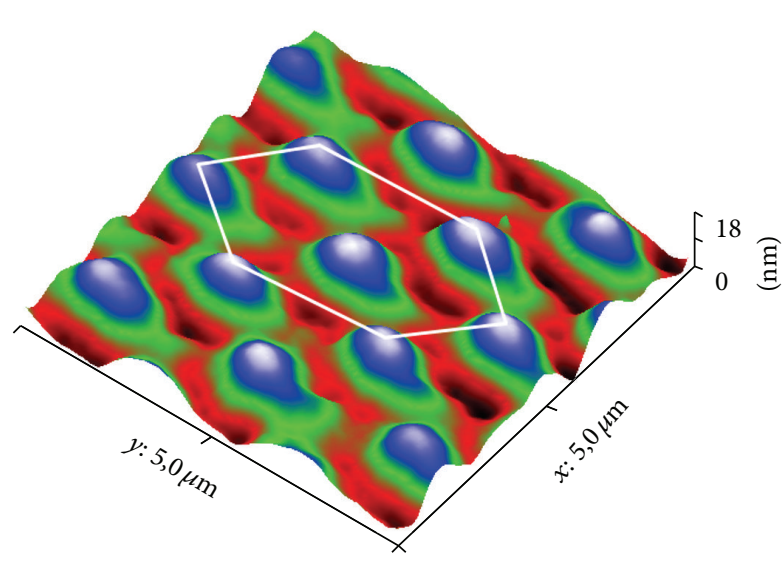

(a)

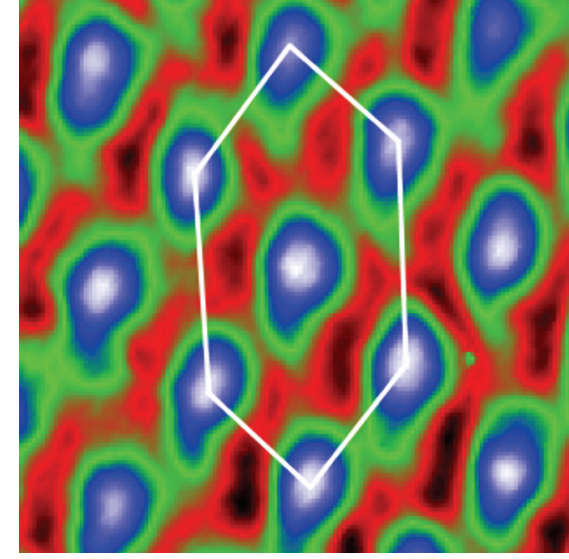

(b)

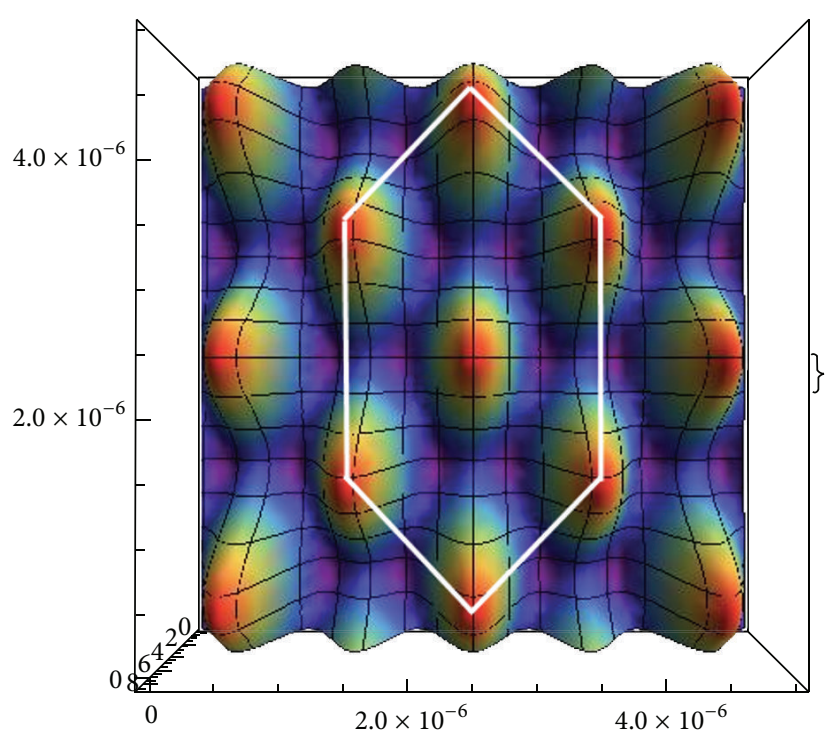

(c)

Figure 8: (a, b) AFM pictures of three-beam holographic recording in $\mathrm{As}_{4} \mathrm{~S}_{1.5} \mathrm{Se}_{4.5}$ sample and (c) its comparison with the theoretical light interference intensity distribution. In this case all three beams are with the same polarization direction; hexagon shaped structure is highlighted in each picture.

polarization stands out from theoretical interference pattern calculations; the light intensity contrast is very close to zero and the electric field intensity gradient plays the main role for the all-optical surface patterning. The behavior of mass transfer and thus the resulting recording could be related to interaction between the polar photo-induced defects with enhanced or decreased polarizability and their drift under the electrical field intensity gradient. A new and relatively easy method has been introduced to investigate the active recording depth or the optimal film thickness which allows optimizing direct recording process and analyzing performance of the specific recording setup. Multibeam holographic recording experiments and their theoretical intensity distribution have been obtained. By using this method it is possible to achieve structures with high geometric precision and by analyzing its theoretical model it is possible to adjust the necessary intensity distribution. There is no doubt that the obtained knowledge and technology can find a practical use in optics (holography, lithography, antireflection coatings, solar batteries, etc.) and electronics (micro- and nanomatrix design), as well as in nanotechnologies (top-down approach) and in many other areas.

\section{Conflict of Interests}

The authors declare that there is no conflict of interests regarding the publication of this paper.

\section{Acknowledgment}

This work has been supported by the European Social Fund within the Project no. 2013/0045/1DP/1.1.1.2.0/13/APIA/ VIAA/018. 


\section{References}

[1] S. K. Tripathy, N. K. Viswanathan, S. Balasubramanian, and J. Kumar, "Holographic fabrication of polarization selective diffractive optical elements on azopolymer film," Polymers for Advanced Technologies, vol. 11, no. 8-12, pp. 570-574, 2000.

[2] P. Rochon, E. Batalla, and A. Natansohn, "Optically induced surface gratings on azoaromatic polymer films," Applied Physics Letters, vol. 66, no. 2, pp. 136-138, 1995.

[3] R. J. Stockermans and P. L. Rochon, "Narrow-band resonant grating waveguide filters constructed with azobenzene polymers," Applied Optics, vol. 38, no. 17, pp. 3714-3719, 1999.

[4] J. Paterson, A. Natansohn, P. Rochon, C. L. Callender, and L. Robitaille, "Optically inscribed surface relief diffraction gratings on azobenzene-containing polymers for coupling light into slab waveguides," Applied Physics Letters, vol. 69, no. 22, pp. 3318-3320, 1996.

[5] C. Egami, Y. Kawata, Y. Aoshima et al., "Two-stage optical data storage in azo polymers," Japanese Journal of Applied Physics, vol. 39, no. 3, pp. 1558-1561, 2000.

[6] L. A. Ibbotson, A. Demetriadou, S. Croxall, O. Hess, and J. J. Baumberg, "Optical nano-woodpiles: large-area metallic photonic crystals and metamaterials," Scientific Reports, vol. 5, article 8313, 2015.

[7] J. Lutkenhaus, F. A. Farro, D. George et al., "Holographic fabrication of 3D photonic crystals using silicon based reflective optics element," Optical Materials Express, vol. 2, no. 9, pp. 12361241, 2012.

[8] Y. Ono and M. Notsu, "Holographic fabrication and transmittance analysis of threedimensional photonic crystals," in Proceedings of the Holography: Advances and Modern Trends II, vol. 8074 of Proceedings of SPIE, The International Society for Optical Engineering, May 2011.

[9] H. Peng, M. Ni, S. Bi, Y. Liao, and X. Xie, "Highly diffractive, reversibly fast responsive gratings formulated through holography," RSC Advances, vol. 4, no. 9, pp. 4420-4426, 2014.

[10] V. Dumarcher, L. Rocha, C. Denis et al., "Polymer thin-film distributed feedback tunable lasers," Journal of Optics A: Pure and Applied Optics, vol. 2, no. 4, pp. 279-283, 2000.

[11] L. Rocha, V. Dumarcher, C. Denis, P. Raimond, C. Fiorini, and J.-M. Nunzi, "Laser emission in periodically modulated polymer films," Journal of Applied Physics, vol. 89, no. 5, pp. 3067-3069, 2001.

[12] D. Bäuerle, Laser Processing and Chemistry, Springer, Berlin, Germany, 2000.

[13] N. Dahotre, Lasers in Surface Engineering, vol. 1 of Surface Engineering Series, ASM International, Materials Park, Ohio, USA, 1998.

[14] J. C. Ion, Laser Processing of Engineering Materials: Principles, Procedure and Industrial Applications, Elsevier ButterworthHeinemann, Oxford, UK, 2005.

[15] W. M. Steen, Laser Material Processing, Springer, London, UK, 3rd edition, 2003.

[16] M. Reinfelde, R. Grants, and J. Teteris, "Photoinduced mass transport in amorphous As-S-Se films," Phys. Status Solidi C, vol. 9, no. 12, pp. 2586-2589, 2012.

[17] U. Gertners and J. Teteris, "Photo-induced mass transfer in chalcogenides," IOP Conference Series: Materials Science and Engineering, vol. 23, no. 1, Article ID 012007, 2011.

[18] G. G. Wells, N. Sampara, E. E. Kriezis, J. Fyson, and C. V. Brown, "Diffraction grating with suppressed zero order fabricated using dielectric forces," Optics Letters, vol. 36, no. 22, pp. 4404-4406, 2011. 

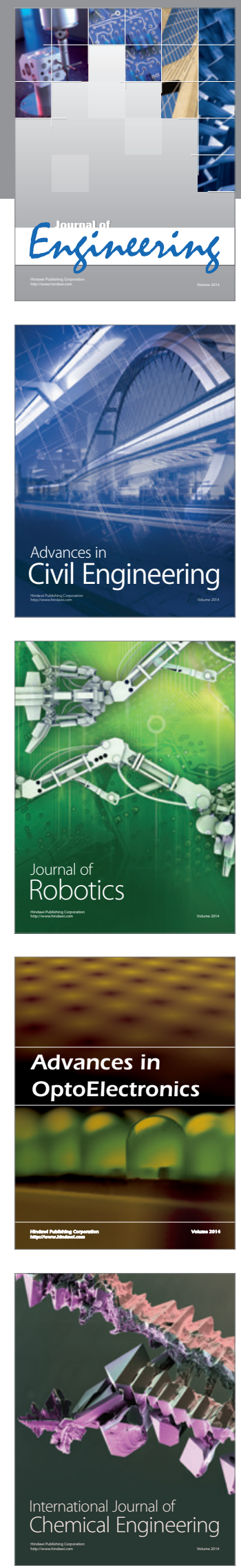

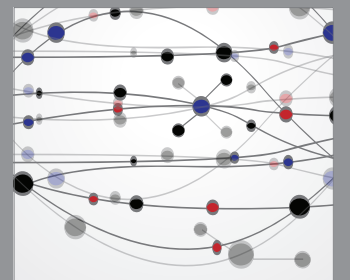

The Scientific World Journal
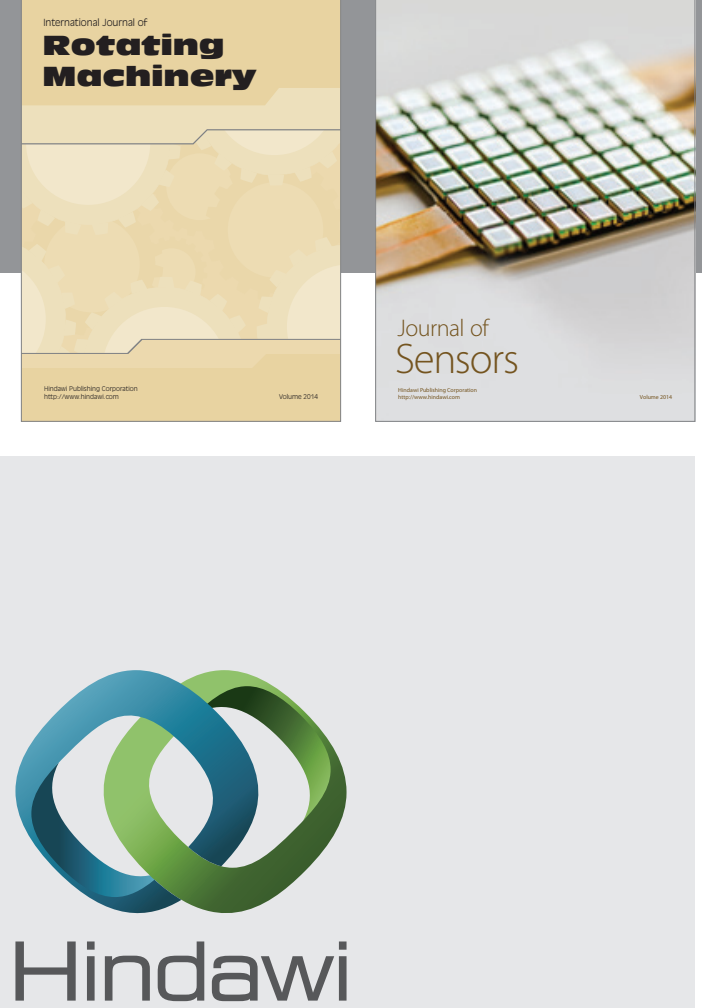

Submit your manuscripts at http://www.hindawi.com
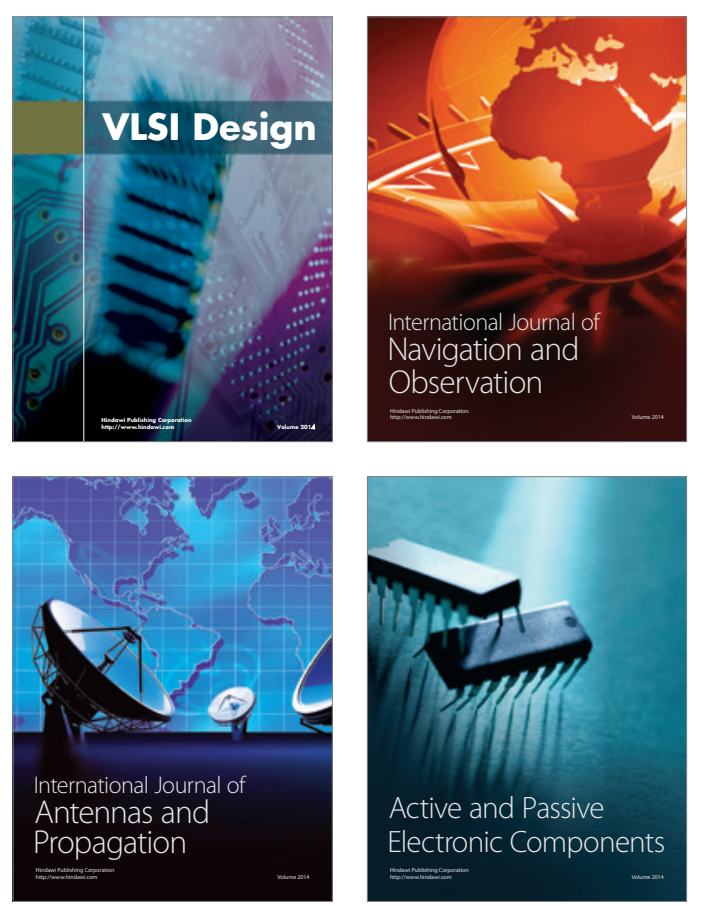
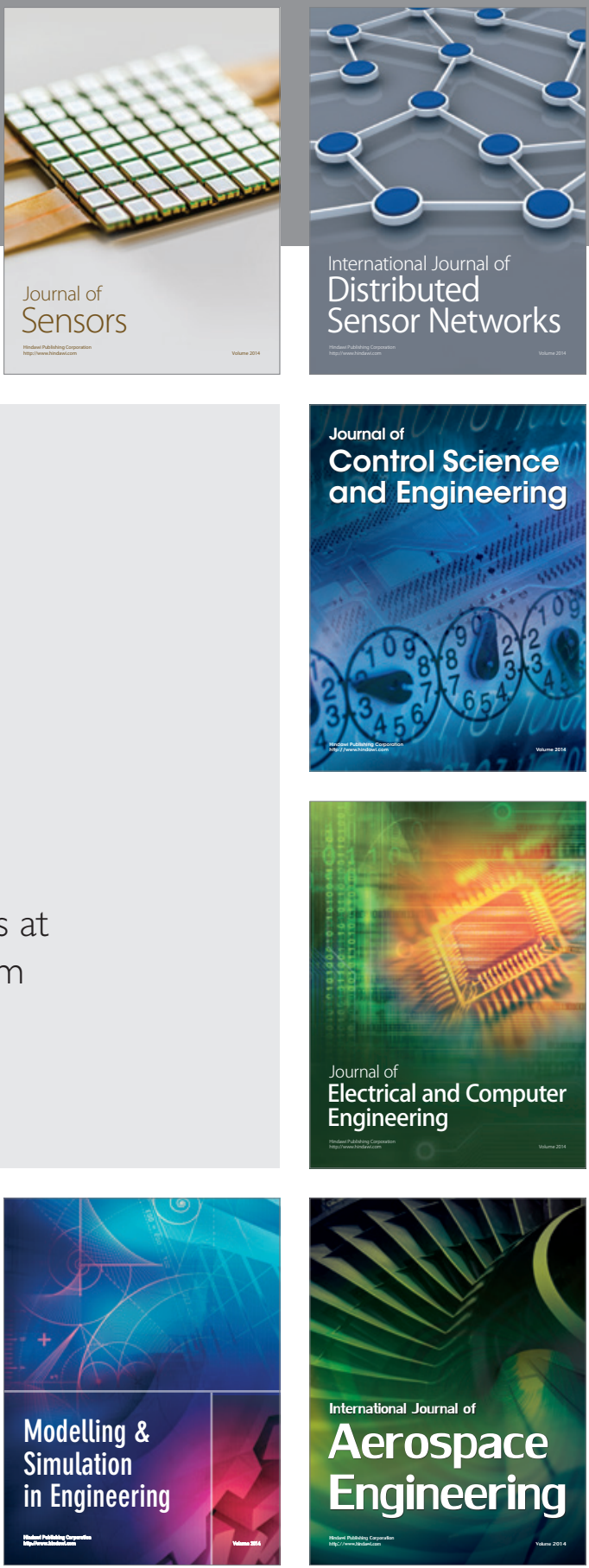

Journal of

Control Science

and Engineering
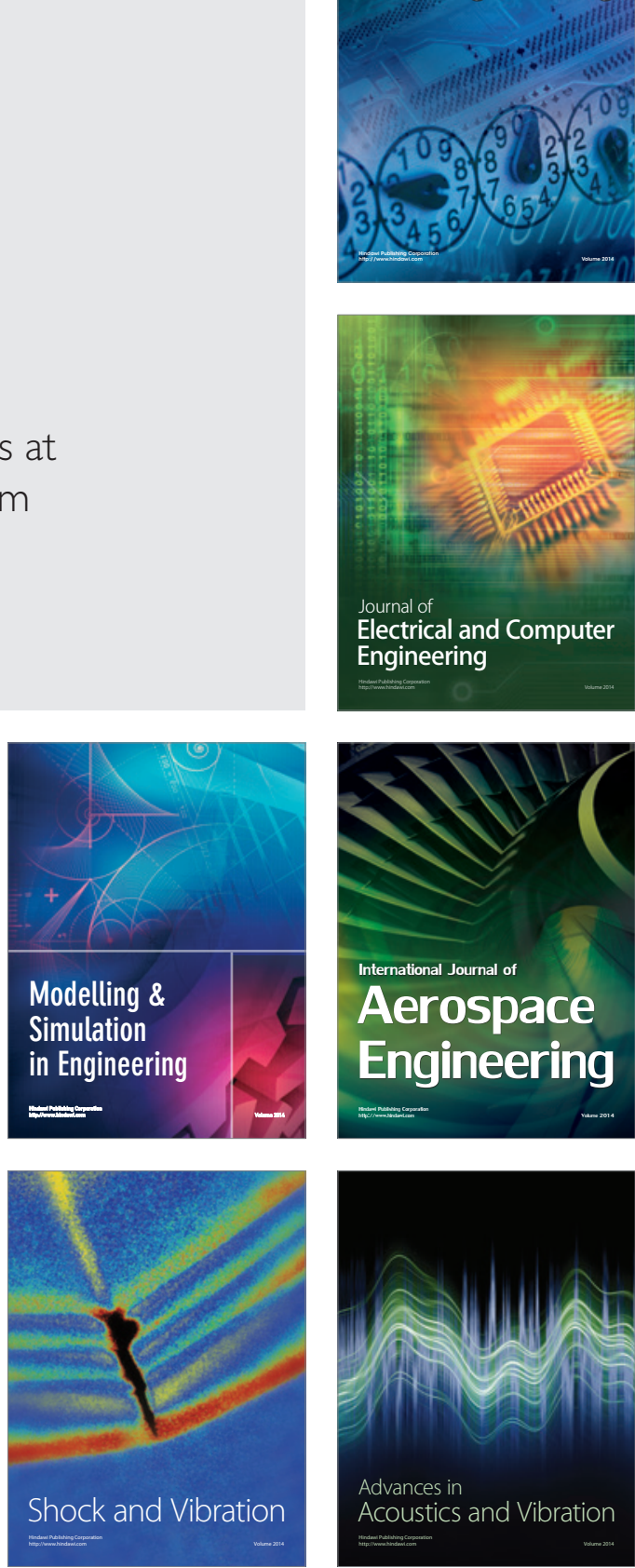